\title{
Risky choice: An examination of information acquisition behavior
}

\author{
JOHN W. PAYNE \\ Graduate School of Business Administration, Duke University, Durham, North Carolina 27706
}

and

\author{
MYRON L. BRAUNSTEIN \\ School of Social Sciences, University of California, Irvine, California 92664
}

\begin{abstract}
The monitoring of information acquisition behavior, along with other process tracing measures such as response times, was used to examine how individuals process information about gambles into a decision. Subjects indicated preferences among specially constructed three-outcome gambles. The number of alternatives available was varied across the sets of gambles. A majority of the subjects processed information about the gambles in ways inconsistent with compensatory models of risky decision making, such as information integration (Anderson \& Shanteau, 1970). Furthermore, the inconsistency between observed information acquisition behavior and such compensatory rules increased as the choice task became more complex. Alternative explanations of risky choice behavior are considered.
\end{abstract}

A large amount of research has been concerned with how individuals use the basic dimensions of information about a gamble-probability of winning (PW), amount to win (\$W), probability of losing (PL), and amount to lose (\$L)-to reach a risky decision (cf. Payne, 1973; Slovic, Fischhoff, \& Lichtenstein, 1977). Most of that research has focused on data that reflect only the end product of the decision processes, for example, choice proportions, rankings, or ratings. In contrast, the present study will use the monitoring of information acquisition behavior, along with other process tracing measures such as response times, to explore the psychological processes used by a subject in arriving at a decision under risk. The collection of such predecisional data seems essential if we are seriously interested in understanding the cognitive processes involved in decision making (Pitz, 1976; Simon, 1976).

The principal theoretical approaches to risky decision making have not included explicit predictions about the information acquisition behavior of the decision maker. It is reasonable to expect, however, that the acquisition of information in a risky decision situation is related to its utilization. The predominant rules proposed for information utilization in risky decision making have assumed a compensatory or tradeoff process. An example of such a rule is the information integration

This research was supported by Research Grant R03MH27588 from the National Institute of Mental Health. We are indebted to Ken Stern for his assistance in the conduct of the experiment and to Steve Schacht for his assistance in the analysis of the results. Requests for reprints should be sent to John W. Payne, Graduate School of Business Administration, Duke University, Durham, North Carolina 27706. model of Anderson and Shanteau (1970). The information integration rule is expressed by the following equation: $R=W_{w} S_{w}+W_{L} S_{L}$, where $R$ is the theoretical response and $S_{w}$ and $S_{L}$ are the basic pieces of information in a risky decision. Anderson and Shanteau argue that this information corresponds to subjective values of the risk dimensions $\$ W$ and $\$ L . W_{w}$ and $W_{L}$ represent measures of the importance of the sources of information to the response. The weights are assumed to be subjective functions of the probabilities, $\mathrm{PW}$ and $\mathrm{PL}$.

As Anderson and Shanteau (1970) point out, their model is similar in form to the subjectively expected utility model that has often served as a normative standard against which behavior could be compared (Slovic et al., 1977). The weights in the integration model, however, are more general than subjective probabilities and do not have to sum to 1.0. In addition, Anderson and Shanteau have stressed that the fundamental purpose of the information integration model is to describe the human thought processes involved in risky decision making, not to prescribe them. A similar model is part of Kahneman and Tversky's (in press) prospect theory of risky choice.

As a model of psychological processes, the information integration model and similar models imply that choice among gambles should proceed as follows: Each gamble in a choice set is evaluated separately. For each gamble, the probability of winning and the amount to win are evaluated (multiplicatively) and then the probability of losing and the amount to lose are evaluated (multiplicatively), or vice versa. The evaluations of the win and lose components of the gamble are then combined into an overall value using an additive rule, or some simple variant (e.g., averaging, Shanteau, 
1975). Comparisons are then made among the overall values of the gambles and the one with greater value is chosen.

The information integration model and similar models are not explicit about how decision processes would relate to information acquisition behavior. Three very plausible implications, however, can be identified. First, the decision maker would most likely use an interdimensional search strategy. That is, search and evaluation would be within a gamble and across dimensions. Such a strategy is in contrast to the intradimensional strategy that has been observed in several studies of decision making (e.g., Bettman \& Jacoby, 1976; Payne, 1976; Russo \& Dosher, Note 1). Second, the decision maker would process the information in a certain order. Specifically, the information integration model proposed by Anderson and Shanteau (1970) would appear to imply that the acquisition of an item of information about a probability dimension would be followed by the acquisition of an item of information about an amount dimension, or vice versa. In contrast, a decision maker might process both probability dimensions and then both amount dimensions. Payne and Braunstein (1971) proposed a model of risky choice that assumed a subject would evaluate both probability dimensions within a gamble before evaluating additional information. Third, a compensatory decision process, such as the information integration model, would imply a constant amount of information search per alternative. On the other hand, a variable amount of search for information across alternatives has been shown to be consistent with certain heuristic noncompensatory decision strategies (Payne, 1976). For example, the elimination-by-aspects model (Tversky, 1972) implies both a variable and an intradimensional pattern of search.

In summary, the present study will examine the processing implications of the information integration model, and those of other risky decision rules, using information acquisition data, along with other process measures such as response times.

\section{METHOD}

\section{Subjects}

Twenty-five college-age subjects were paid at a fixed hourly rate for their participation in the experiment. In addition, each subject was given an initial stake to be used in playing selected gambles (see Procedure). The subjects were naive with respect to the task and stimuli.

\section{Stimuli}

The stimuli were 12 sets of three-outcome gambles. Within the 12 sets of gambles, 4 sets contained two gambles, 4 contained four gambles, and 4 contained eight gambles. The number of choice alternatives available has been shown to affect the likelihood that subjects will use compensatory as opposed to noncompensatory decision processes (Payne, 1976). Each gamble in a set involved a probability of winning (PW), an amount of money to win (\$W), a probability of losing (PL), an amount of money to lose (\$L), and a probability of neither a win nor a loss $(P Z)$, such that $P W+P L+P Z=1 . P Z$ was not displayed to the subjects. PW and PL varied from .05 to .8 in increments of .05 . $\$ W$ and $\$ L$ varied from $\$ .05$ to $\$ .80$ in increments of $\$ .05$. Since the probability relationship within a gamble in a choice set has been found to be related to preference judgments in several studies (Payne, 1975; Payne \& Braunstein, 1971; Ranyard, 1976), half the choice sets had PW $>$ PL for all gambles, and half the sets had PW $<$ PL for all gambles. Finally, the four two-gamble sets, two of the fourgamble sets, and two of the eight-gamble sets had equal expected values. Two of the four-gamble sets and two of the eight-gamble sets had unequal expected values.

The stimuli were displayed on a computer terminal (Digital Equipment Corporation VT05) connected on-line to a PDP-11 computer. The gambles were displayed in a matrix format on the screen of the terminal. For half of the choice sets, rows in the matrix represented the gambles $(A, B, \ldots)$ and the columns represented the four risk dimensions. For the remaining choice sets, the rows represented the risk dimensions and the columns represented the gambles. The order in which the risk dimensions were presented was randomized across trials. The letter associated with a particular gamble in each of the 12 choice sets was randomized across subjects.

A row of keys on the computer terminal was labeled I, II, $\ldots$, VIII, referring to the columns of the display matrix, and a second row of keys was labeled $1,2, \ldots, 8$, referring to the rows of the display matrix. In addition, there was a key labeled "information" and a key labeled "choice."

The subjects were instructed that they could obtain information about any particular alternative on any particular risk dimension by pressing the key corresponding to the row and the key corresponding to the column of the cell that they wished to check and then by pressing the "information" key. The subjects were told that on selecting a new item of information, the previous item would be erased, but that they could go back and recheck an item if they wished. This procedure provides data resembling that acquired by an eye movement procedure (e.g., Russo \& Dosher, Note 1). However, the procedure allows for a relatively more complex decision problem to be displayed to the subjects than those used in eye movement studies.

\section{Procedure}

The subjects were tested individually in two separate 1-h sessions occurring on consecutive days. In the first session, the subject received one practice problem and six additional decision problems. The second session consisted of six more decision problems. In each session, the subject always received two two-, four-, and eight-gamble decision problems. Each session always included a mixture of choice sets with gambles PW $>$ PL and choice sets $\mathrm{PW}<\mathrm{PL}$. Within these these constraints, the order of presentation was randomized across sessions and subjects.

The subjects were instructed to choose the gamble they would most prefer to play. A three-outcome gamble was explained and a sample gamble demonstrated. The subject was instructed in the information acquisition procedure and told to decide which gamble was preferred and then indicate the selection using the "choice" key.

Five of the 25 subjects were selected on a random basis to give verbal protocols. The instructions were to "think aloud" continuously as they selected their preferred gambles in each decision situation.

In order to increase motivation and provide real consequences for the decisions, the subjects were given a $\$ 1.50$ initial stake and told that at the end of the session two of their preferred gambles would be chosen and played, and that they would be allowed to keep the money they had after playing the gambles. Finally, the subjects were told to work at their own pace and that there should be plenty of time to finish. 
During the experiment, the order in which information was examined, the amount of time between information requests (search time), the time between the last information request and the subjects' indication of a choice (decision time), and the gamble chosen were recorded. The reaction times were recorded to an accuracy of $.03 \mathrm{sec}$.

\section{RESULTS}

\section{Response Times and the Depth and Sequence of Search}

The search data for each subject were first organized in terms of the percentage of the total available information searched in each decision situation, the absolute value of the differences between the percentage of information searched per gamble and the mean percentage of total information searched for each situation (reflecting the depth of search across gambles), and the sequence of search (interdimensional or intradimensional). The measure of variation in depth of search across gambles was the same as that used by Payne (1976) to allow comparison between search behavior in a risky and nonrisky choice task. The index of sequence of search was determined by examining the gamble and dimension associated with the $n+1$ th piece of information searched by a subject as a function of the nth piece of information searched. If the $n+1$ th piece of information searched was within the same gamble but involved a different dimension, then that constituted an instance of an interdimensional sequence of search. On the other hand, if the $n+1$ th piece of information searched was within the same dimension, but a different gamble, then that constituted an instance of an intradimensional sequence of search. If the $n+1$ th piece of information searched was within neither the same gamble nor the same dimension on the $n$th piece of information, then that was considered to be a shift in the sequence of information search. The measure of search sequence for a particular situation was determined by the number of interdimensional single-step transitions minus the number of intradimensional singlestep transitions divided by the sum of the two numbers. A sequence consisting only of interdimensional transitions and shift transitions would have a value of +1.00 . A sequence consisting only of intradimensional transitions and shift transitions would have a value of -1.00 . Again, this index of search was similar to that used by Payne (1976), in order to allow comparisons across studies. It should be noted, however, that this index, unlike Payne's (1976) index, is based on both the original acquisition of a piece of information and repeated examinations of that piece of information. Table 1 presents the mean percentage of the total available information searched, the mean variation in information search per gamble, and the mean value of the search sequence index, across the 25 subjects for the two-, four-, and eight-gamble choice sets. Also presented in Table 1 are the mean search times and decision times in seconds.

A multivariate analysis of variance was conducted to determine the effects of variation in number of gambles available on the five dependent variables presented in Table 1. The main effect of number of gambles (two vs. four, eight, and four vs. eight) was significant $[F(10,232)=7.25, p<.01]$. To more fully identify the effects of number of gambles, separate univariate analyses of variance were conducted for each of the dependent variables. The effect of the number of gambles on the proportion of total available information searched was significant $[F(2,120)=27.19, p<.01]$. The proportion of information searched decreased as the number of gambles increased. The main effect of number of gambles on the amount of variation in information searched per gamble was also significant $[F(2,120)=15.82, p<.01]$. The amount of variation in information searched per gamble increased as the number of gambles available in the choice set increased. This increase is consistent with the results obtained by Payne (1976), but the amount of variation in search per gamble was less than the variation in search found by Payne in situations involving choice among apartments. Nevertheless, the result indicates that some gambles were eliminated after only a limited amount of search. The tendency across subjects to employ a decision strategy consistent with compensatory models such as information integration (Anderson \& Shanteau, 1970) appears to decrease as the number of gambles available increases. The main effect of the number of gambles on sequence of search was significant $[F(2,120)=4.61, p<.05] .{ }^{1}$ There was more intradimensional search as the number of gambles increased. The analyses of the response time measures were mixed. The mean search time decreased as the number of gambles increased $[F(2,120)=4.72$, $p<.05]$. Decision time also appeared to decrease as the number of gambles increased, but the effect was not significant $[F(2,120)=1.64]$.

The mean values across subjects and gambles for each of the five dependent variables in Table 1 were also calculated as a function of the probability of winning/

Table 1

Means for the Depth and Sequence of Information Search Measures and for the Response Times

\begin{tabular}{cccccc}
$\begin{array}{c}\text { Number of } \\
\text { Alternatives }\end{array}$ & $\begin{array}{c}\text { Percentage of Infor- } \\
\text { mation Searched }\end{array}$ & $\begin{array}{c}\text { Variation in Search } \\
\text { per Alternative }\end{array}$ & $\begin{array}{c}\text { Pattern of } \\
\text { Search }\end{array}$ & $\begin{array}{c}\text { Search } \\
\text { Time }\end{array}$ & $\begin{array}{c}\text { Decision } \\
\text { Time }\end{array}$ \\
\hline 2 & .92 & .01 & .32 & 9.92 & 25.33 \\
4 & .83 & .06 & .19 & 8.89 & 20.77 \\
8 & .76 & .08 & .13 & 8.64 & 19.98 \\
\hline
\end{tabular}


probability of losing relationships within the gambles in a choice set. A two-way multivariate analysis of variance (probability. relationship within gambles, and subjects) showed no significant effect $[F(5,116)=1.41]$.

Finally, the interaction of the probability relationship with number of gambles available was not significant $[F(10,232)=.56]$.

\section{The Content of Search}

The search data for each subject were next organized in terms of information acquisition across the four basic risk dimensions, PW, $\$ \mathrm{~W}, \mathrm{PL}$, and $\$ \mathrm{~L}$. That is, the type of information typically examined first, the type of information examined second, and so on, was determined for each subject (content of search). There are 24 possible rank orders in which the four risk dimensions can be examined. These 24 rank order patterns can be divided into three types: One type (eight patterns) involves the search for information about the probabilities, either interdimensionally or intradimensionally, and then amounts, or vice versa. A second type (eight patterns) involves the acquisition of information about the win component of the gambles (PW and \$W) and then the loss component (PL and $\$ \mathrm{~L}$ ), or vice versa. Again, the acquisition of information might be either interdimensional or intradimensional. Finally, a subject could acquire information about one risk dimension (e.g., PW), followed by information about a second risk dimension (e.g., $\$ \mathrm{~L}$ ) that is not of the same type (probabilities or amounts) and does not refer to the same component (win or loss) of a gamble (eight patterns).

A classification of the 300 search patterns exhibited by the subjects ( 25 subjects by 12 treatment combinations) into one of the three types of dimensional search patterns showed 124 patterns of the probabilitiesamounts type, 158 patterns of the win-loss type, and 18 patterns of neither type. If the subjects were searching the risk dimension information in a random fashion, or just in terms of left-to-right reading order on the computer display, the number of probabilityamount, win-loss, and "neither" types of search patterns would be expected to be equal, since the position of the risk dimensions was randomized across trials. The relatively small number of search patterns in the "neither" category (18 out of 300 ) indicates that the subjects did systematically search for information across the risk dimensions. More interestingly, a substantial number of the search patterns (124 out of 300 ) involved either the processing of information about both probability components of the gambles then the amount components, or the reverse. These 124 search patterns are not consistent with the types of processing implied by an information integration model of risky choice such as that proposed by Anderson and Shanteau (1970). That type of model implies that information is processed either within the win component of a gamble and then within the loss component of a gamble, or the reverse. Combined with the earlier results concerning the depth of search, this result suggests that a number of subjects may have employed processing rules other than those implied by the information integration model and other models specifying similar utilization of the probability and outcome dimensions.

\section{Individual Differences}

So far, our analyses of the information search and response time measures have focused on data grouped across subjects. Information processing psychology, however, from which process tracing methodologies are derived, stresses the need to pay attention to the behavior of individual subjects (Simon, 1976).

To explicate possible individual differences in risky decision making, each of the 25 subjects was classified into 1 of 12 categories of search behavior, depending on whether the pattern of search exhibited by the subject was primarily probabilities-amounts, win-loss, or neither, whether the search pattern was primarily interdimensional or intradimensional, and whether the search patterns indicated that the variation in amount of information searched per alternative increased as the number of alternatives available increased. The classification of a subject as processing in a probabilities-amounts, win-loss, or neither fashion was determined by calculating the number of patterns for the subject that were of a probabilities-amounts type, minus the number of patterns of a win-loss type, divided by the sum of the two numbers. If the value of that number was positive, the subject was classified as processing primarily in a probabilities-amounts fashion (10 subjects). If the value was negative, the subject was classified as processing the win-loss components of the gambles (13 subjects). The means of this measure for the subjects classified as probabilities-amounts, win-loss, or neither (two subjects) were $.87,-.65$, and 0 . The classification of a subject as processing in either an interdimensional or an intradimensional fashion was based on the mean sequence index for the subject. If the value of the sequence index was positive, the subject was classified as processing primarily in an interdimensional fashion (16 subjects). If the index was negative, the subject was classified as processing primarily in an intradimensional fashion (nine subjects). The mean search indexes for the subjects classified as interdimensional and intradimensional were .45 and $-.27 .^{2}$ Finally, the mean amount of variation in information searched per alternative was determined for each subject as a function of the number of alternatives available. The mean amounts of variation for those subjects classified as showing an increased variation in amount of search for the two-, four-, and eight-alternative choice situations (14 subjects) were $.01, .10$, and .12 . The mean amounts of variation for those subjects classified as not showing an increase for the two-, four-, and eight-alternative choice situations (11 subjects) were $.02, .02$, and .03 . The classification 
Table 2

Classification of Information Search Pattern for Each Subject

\begin{tabular}{|c|c|c|c|c|}
\hline \multirow{3}{*}{$\begin{array}{c}\text { Content of Search Across } \\
\text { Risk Dimensions }\end{array}$} & \multirow{2}{*}{\multicolumn{2}{|c|}{$\frac{\text { Interdimensional Sequence of Search }}{\text { Depth of Search }}$}} & \multirow{2}{*}{\multicolumn{2}{|c|}{$\frac{\text { Intradimensional Sequence of Search }}{\text { Depth of Search }}$}} \\
\hline & & & & \\
\hline & Constant & Variable & Constant & Variable \\
\hline Probabilities/Amounts & & $\begin{array}{l}\text { Subject } 3 \\
\text { Subject } 10 \\
\text { Subject } 12 \\
\text { Subject } 24 \\
(1.82,1.63,1.40)\end{array}$ & $\begin{array}{l}\text { Subject } 15 \\
(1.10,2.86,4.44)\end{array}$ & $\begin{array}{l}\text { Subject } 7 \\
\text { Subject } 16 \\
(.94,2.41,4.24)\end{array}$ \\
\hline Win/Loss & $\begin{array}{l}\text { Subject } 8 \\
\text { Subject } 11 \\
\text { Subject } 14 \\
\text { Subject } 18 \\
\text { Subject } 19 \\
\text { Subject } 20 \\
\text { Subject } 25 \\
(2.77,2.81,2.84)\end{array}$ & $\begin{array}{l}\text { Subject } 2 \\
\text { Subject } 13 \\
(1.71,1.77,1.60)\end{array}$ & $\begin{array}{l}\text { Subject } 22 \\
(.50,3.50,7.25)\end{array}$ & $\begin{array}{lr}\text { Subject } & 1 \\
\text { Subject } & 4 \\
\text { Subject } & 6 \\
\text { Subject } & 23 \\
(.99,2.44,4.40)\end{array}$ \\
\hline Neither & $\begin{array}{l}\text { Subject } 9 \\
(2.73,2.14,2.44)\end{array}$ & & & $\begin{array}{l}\text { Subject } 5 \\
(1.06,2.34,4.07)\end{array}$ \\
\hline
\end{tabular}

Note-The numbers in parentheses are the average lengths of the search sequences for the subjects in each of the 12 categories for the two-, four-, and eight-alternative choice situations, respectively.

of each of the 25 subjects into these 12 categories is given in Table 2.

The eight subjects classified as having primarily winloss, interdimensional, and constant patterns of search appear to be individuals whose behavior is consistent with an information integration, or perhaps expectation, type of model. Additional support for the view that these eight subjects used those types of processes was obtained by calculating the average length of an interdimensional sequence of processing. A strict expected value process, for example, should show a sequence of three interdimensional single-step transitions, that is, probability to win of Gamble A, followed by amount to win of Gamble A, followed by probability to lose of Gamble A, followed by amount to lose of Gamble A. Furthermore, the average length of the sequence should not vary as a function of the number of alternatives available. The average length of sequence for these eight subjects for the two-, four-, and eight-altemative choice situations was $2.77,2.81$, and 2.84 . Only one subject, Subject 14, showed inconsistent sequence lengths of $1.83,1.18$, and 1.83 for the two-, four-, and eight-alternative situations.

Although the focus of the present study was not on the final choice responses, it is also interesting to compare the final choices made by the eight subjects with information integration or expectation types of search patterns with the choices of the other 17 subjects. Each subject in this study faced four situations that contained sets of gambles with unequal expected values (see Stimuli). For the eight subjects with information integration or expectation types of search patterns, the mean numbers of choices consistent with the maximization of expected value was 3.13 . The mean number of choices consistent with the maximization of expected value for the other 17 subjects in the study was 1.30 . A test of the difference between the two means was significant $[t(23)=4.36, \quad p<.01]$. This apparent agreement between a standard measure of the end product of the decision process and the measures of predecisional behavior serves to validate the methodology and classification of subjects employed in this study.

Next, consider the six subjects who exhibited primarily intradimensional and variable patterns of search involving either probabilities-amounts or win-loss. Payne (1976) suggested that individuals with an intradimensional sequence of search and increasing variation in amount of information search may be using an additive difference type process to select among two alternatives and perhaps an elimination-by-aspects type process to select among multiple alternatives. An examination of the average length of the sequences of intradimensional processing for the six subjects classified as having intradimensional and variable search patterns given in Table 2 supports this view: As the number of alternatives available increases, the average length of an intradimensional processing sequence increases. The fact that the average length of sequence for the four- and eight-alternative choice situations is substantially larger than 1.0 indicates that these subjects were not using the sort of standard revision version of the additive difference model suggested by Russo and Rosen (1975) as a way of dealing with a multialternative choice problem. Instead, the length of the sequences of intradimensional processing suggests that these subjects may have employed the heuristic intradimensional processing rule elimination by aspects (Tversky, 1972). It is interesting to note that those subjects who were classified as having interdimensional and variable search 
patterns have an average processing sequence length shorter than the subjects who were classified as having interdimensional and constant search patterns. The relatively short sequence of interdimensional processing, together with the variable search pattern, is consistent with either a conjunctive type process or the contingent processing model proposed by Payne and Braunstein (1971).

The classification of subjects presented in Table 2 is based on an analysis of information search across all 12 decisions. However, given the significant effects of number of gambles available on the grouped data presented in Table 1, the search behavior of each subject was further classified as a function of the number of gambles in a choice set. In particular, the search behavior for the simplest choice problems (two gambles) and the most complex choice problems (eight gambles) were compared for each subject. In terms of content of search, the number of subjects classified as win-loss, probabilitiesamounts, or neither went from 15,8 , and 2 (two-gamble sets), to 12,9 , and 4 (eight-gamble sets). This suggests that the content of search remained essentially the same in the eight-gamble sets as it was in the two-gamble sets. In terms of depth of search, however, the number of subjects classified as constant or variable went from 24 and 1 to 10 and 15 . Considering only those subjects who shifted from one classification to another, 14 subjects went from constant to variable, and no subjects went from variable to constant $(p<.01)$. This result for individual subjects is consistent with the analysis of variance results and indicates that decision strategies shifted as a function of the complexity of the decision task. In terms of sequence of search, the number of subjects classified as interdimensional or intradimensional shifted from 20 and 5 for the two-gamble sets to 13 and 12 for the eight-gamble sets. Again considering only those subjects who shifted from one classification to another, eight subjects went from interdimensional to intradimensional, and one subject shifted in the other direction $(\mathrm{p}<.05)$.

\section{DISCUSSION}

An analysis of the patterns of information search exhibited by decision makers demonstrated that information about gambles is often processed in ways inconsistent with the information acquisition implications of compensatory models of risky decisions, such as information integration theory. In particular, subjects often searched for information in terms of the two probability dimensions and then the two amount dimensions and not in terms of the probability to win and amount to win and then the probability to lose and amount to lose. Furthermore, the tendency for decision makers to adopt other decision strategies increased as the number of gambles in a choice set increased. This was shown most clearly by the increase in the variation in amount of search per gamble as the choice-set size was increased. Such a result is consistent with previous experiments on preferential choice in nonrisky situations and supports the idea that decision makers resort to choice heuristics as the task becomes more complex (Payne, 1976).

The present study also demonstrated the existence of large individual differences in the ways in which information about gambles was processed. For example, some of the subjects tended to acquire information about the two probability values and then about the two amounts, or the reverse. Other subjects tended to acquire information about the win component of a gamble and then about the loss component, or the reverse. The reasons for this individual difference is not clear. The individuals who processed information in terms of the wins and losses may have been using the probabilities as weights to be applied to the outcomes of the gambles. This would be consistent with the information integration model or a similar model. It is also possible that some of the subjects may have possessed statistical knowledge that would have led to an expectation approach to decision making. Some support for such an effect has been found by Schoemaker (1977). He found a positive relationship between the amount of statistical training of undergraduates and the extent to which bids for gambles were consistent with expected values. On the other hand, Lichtenstein, Slovic, and Zink (1969) found that telling people about the expected value concept did not lead to a significant increase in the use of that concept as a guide to action. More research is needed on how knowledge already possessed by a decision maker and other subject characteristics influence decision processes.

The reasons for the processing of the probability to win and the probability to lose together and then the amount to win and the amount to lose together are also not clear. One possible explanation relates to the issue of dimensional commensurability raised by Slovic and MacPhillamy (1974). It may be that comparing the two probability dimensions of a gamble and then comparing the two amount dimensions is easier than attempting to integrate probability information with amount information separately for the win and loss components. In other words, the "chunking" process may be easier for commensurate dimensions. This explanation is only speculative, but it does suggest an important question: What happens as the complexity of a risky alternative is increased through increases in the number of outcomes and probabilities? One possibility would be for the decision maker to treat all outcomes below a certain level as similar. That is, a decision maker might establish a target level of return (Fishburn, 1977) and combine all the probabilities associated with outcomes below that level into one composite probability of failure to meet the aspiration level. The manner in which complex gambles are reduced to simpler ones, however, 
is yet to be investigated (Kahneman \& Tversky, in press). Another important individual difference was whether a subject tended to process information in an interdimensional or in an intradimensional fashion. This sort of difference has appeared in several studies of individual search behavior (e.g., Bettman \& Jacoby, 1976; Payne, 1976; Russo \& Dosher, Note 1). A possible explanation for this difference has been offered in terms of individual differences in how the decision maker represents the knowledge he or she acquires about the alternatives in a decision task (Payne, 1976).

It is also interesting to note that the amount of interdimensional processing observed in the present risky choice experiment was greater than that observed in the studies involving choices among apartments (Payne, 1976) and choices among cold breakfast cereals (Bettman \& Jacoby, 1976). This result is consistent with the finding by Russo and Dosher (Note 1) that the relative amount of interdimensional or holistic processing was greatest for their experiment involving binary choice among very simple gambles, as opposed to graduate school applicants. Rosen and Rosenkoetter (1976) have obtained similar results. One explanation for this across-study or across-task effect has been offered in terms of the perceived interrelatedness of the information dimensions (Russo \& Dosher, Note 1). Another explanation is in terms of differences in the nature of the information retrieval system available to the decision maker. For example, this study differed from Payne (1976) and Payne and Braunstein (Note 2) in the increased memory load placed on the subjects. In both of the earlier studies, once a particular piece of information had been acquired, it was always clearly visible to the decision maker. The present study did not provide an external memory, and some of the subjects may have tried to form larger chunks of information by combining, for example, the probability to win and the probability to lose into a single chunk of information regarding the odds associated with each gamble. Variations in information display have been shown to affect processing (Bettman \& Kakkar, 1977). Of course, both explanations may be viable. Much more research is needed to determine how the processing of information by a decision maker varies as a function of the information retrieval system being used and as a function of the nature of the choice alternatives.

\section{REFERENCE NOTES}

1. Russo, J. E., \& Dosher, B. A. Dimensional evaluation: A heuristic for binary choice. Unpublished manuscript, University of California, San Diego, 1975.

2. Payne, J. W., \& Braunstein, M. L. Task complexity and contingent processing in decision making: $A$ replication and extension to risky choice. Unpublished manuscript, University of Chicago, 1977.

\section{REFERENCES}

Anderson, N. H., \& Shanteau, J. C. Information integration in risky decision making. Journal of Experimental Psychology, 1970, 84, 441-451.

Bettman, J. R., \& JAcoby, J. Patterns of processing in consumer information acquisition. In B. B. Anderson (Ed.), Advances in consumer research (Vol. 3), 1976, 315-320.

BetTMan, J. R., \& KaKkaR, P. Effects of information presentation format on consumer information acquisition strategies. Journal of Consumer Research, 1977, 3, 233-240.

Fishburn, P. C. Mean-risk analysis with risk associated with below-target returns. The American Economic Review, $1977,67,116-126$.

Kahneman, D., \& Tversky, A. Prospect theory: An analysis of decision under risk. Econometrica, in press.

Lichtenstein, S., Slovic, P., \& Zink, D. Effect of instruction in expected value on optimality of gambling decisions. Journal of Experimental Psychology, 1969, 79, 236-240.

Payne, J. W. Alternative approaches to decision making under risk: Moments vs. risk dimensions. Psychological Bulletin, 1973, 80, 439-454.

PAYNE, J. W. Relation of perceived risk to preferences among gambles. Journal of Experimental Psychology: Human Perception and Performance, 1975, 104, 86-94.

Payne, J. W. Task complexity and contingent processing in decision making: An information search and protocol analysis. Organizational Behavior and Human Performance, 1976, 16, 366-387.

Payne, J. W., \& Braunstein, M. L. Preferences among gambles with equal underlying distributions. Journal of Experimental Psychology, 1971, 87, 13-18.

Pirz, G. F. Decision making and cognition. In H. Jungerman \& G. de Seeuw (Eds.). Proceedings of the fifth research conference on subjective probability, utility, and decision making. Dordrecht, Holland: Reidel, 1976.

RANYARD, R. H. Elimination by aspects as a decision rule for risky choice. Acta Psychologica, 1976, 40, 299-310.

Rosen, L. D., \& Rosenkoetter, P. An eye fixation analysis of choice and judgment with multiattribute stimuli. Memory \& Cognition, 1976, 4, 747-752.

Russo, J. E., \& Rosen, L. D. An eye fixation analysis of multialternative choice. Memory \& Cognition, 1975, 3, $267-276$.

SCHOEMAKer, P. J. H. Experimental studies on individual decision making under risk: An information processing approach. Unpublished doctoral dissertation, University of Pennsylvania, 1977.

Shanteau, J. An information-integration analysis of risky decision making. In M. F. Kaplan \& S. Schwartz (Eds.), Human judgment and decision processes. New York: Academic Press, 1975.

Simon, H. A. Discussion: Cognition and social behavior. In J. S. Carroll \& J. W. Payne (Eds.), Cognition and social behavior. Hillsdale. N.J: Erlbaum, 1976. Pp. 253-267.

Slovic, P., Fischhoff, B., \& Lichtenstein, S. Behavioral decision theory. Annual Review of Psychology, 1977, 28, $1-39$.

Slovic, P., \& McPhillamy, D. Dimensional commensurability and cue utilization in comparative judgment. Organizational Behavior and Human Performance, 1974. 11, 174-194.

TVERSKY, A. Elimination by aspects: A theory of choice. Psychological Review, 1972, 79, 281-299. 


\section{NOTES}

1. Bettman and Jacoby (1976) have suggested a correction to the search index used by Payne (1976) and in this study in order to account for the total number of items of information examined and for the actual number of alternatives and dimensions examined by a subject in a particular choice situation. For the present data, the correlation between the index used and the index with the Bettman correction is .97 The Bettman correction, however, does reduce the means for the index to $.24, .16$, and .14 for the two-, four-, and eight-gamble situations, respectively. The $\mathrm{F}$ value for the univariate analysis of variance is also reduced $[F(2,120)=1.47]$. The multivariate analysis of variance remains significant $[F(10,232)=6.90$, $\mathrm{p}<.01]$

2. The classification of subjects using the search index with the Bettman correction is essentially the same. The only change would be to move the pattern of search classification for Subject 21 from interdimensional to intradimensional.

(Received for publication April 12, 1978; revision accepted August 1, 1978.) 